\title{
Os dados reais versus a divulgação da mídia do perfil de apreensão de drogas ilícitas na região Centro-Oeste de Minas Gerais
}

\author{
F.F. Machado a, A.C. C. Moura ${ }^{\text {a }}$, T.L.S. Sales ${ }^{\text {a }}$, P.L. Lima ${ }^{\text {b }}$, A.C.M. Costa ${ }^{\text {a }}$ C. Sanches ${ }^{\text {a }}$, \\ F.M.D. Chequer ${ }^{\mathrm{a},{ }^{*}}$ \\ ${ }^{a}$ Universidade Federal de São João del-Rei Campus Centro-Oeste Dona Lindu (UFSJ-CCO), Divinópolis (MG), Brasil \\ ${ }^{b}$ Posto de Perícia Integrado, $7^{\circ}$ Departamento de Polícia Civil, Divinópolis (MG), Brasil \\ *Endereço de e-mail para correspondência: farahchequer@ufsj.edu.br.Tel.: +55-37-36904497
}

Recebido em 29/01/2020; Revisado em 09/06/2020; Aceito em 14/06/2020

\begin{abstract}
Resumo
O consumo de drogas é um dos principais problemas de saúde pública. Os dados de apreensões de drogas ilícitas feitas pelas Polícias Federal, Estadual e Municipal fornecem dados indiretos do consumo de drogas e permitem traçar o perfil de uso dessas substâncias em uma determinada população. Este estudo teve como objetivo descrever o perfil de apreensão de drogas ilícitas na região Centro-Oeste de Minas Gerais, comparando as informações disponíveis na mídia com os dados reais fornecidos pela Perícia Criminal da Polícia Civil de Divinópolis-MG. É um estudo observacional descritivo realizado por meio da busca de reportagens que abordassem apreensões de drogas em cidades pertencentes à região Centro-Oeste de Minas Gerais e de dados secundários fornecidos pela Perícia Criminal acerca de Registros de Eventos da Defesa Social envolvendo drogas entre o período de 2017 e 2018. Dos 208 detidos identificados nas reportagens, $73,1 \%$ são do sexo masculino e 10,6\% sexo feminino, com idade média de 21 anos. Dentre as drogas reportadas, a maconha foi a droga mais apreendida seguida pela cocaína e seus derivados. Apesar das maiores apreensões de drogas serem atribuídas pelas reportagens às regionais de Formiga e Bom Despacho, conforme as informações reais da Perícia Criminal, as regionais de Nova Serrana e Pará de Minas foram as com maiores apreensões. O perfil de apreensão de drogas torna-se um referencial importante para subsidiar o planejamento de políticas públicas conforme a real demanda, visando à prevenção do comércio e ao combate ao uso dessas substâncias.
\end{abstract}

Palavras-Chave: Apreensão de Drogas; Drogas Ilícitas; Saúde Pública.

\begin{abstract}
Drug abuse is one of the main public health problems, mainly due to its association with violence, crime and marginality. Data from illicit drug seizures by the Federal, State and Municipal Police provide indirect data on drug use and allow the profile of the use of these substances to be traced in a given population. Thus, this study aimed to describe the profile of illicit drug seizures in the Midwest region of Minas Gerais, comparing information available in the media with real data, allowed by Divinópolis-MG Civil Police Criminal Expertise. It's a descriptive observational study carried out through the search of reports that approached drug seizures in cities belonging to the Central-West region of Minas Gerais and secondary data provided by the Criminal Expertise about Social Defense Event Records involving drugs between 2017 and 2018. Of the 208 detainees identified in the reports, $73.1 \%$ are male and 10.6\% female, with a mean age of 21 years. Among the drugs reported, marijuana was the most seized drug followed by cocaine and its derivatives. Although the largest drug seizures are attributed by the reports to the Regional Ant and Good Dispatch, according to the actual information of the Criminal Forensics, the regions of Nova Serrana and Pará de Minas are the ones with the largest seizures. The drug seizure profile of a given region becomes an important reference point to support the planning of public policies according to the real demand, aiming at preventing trade and fighting the use of these substances.
\end{abstract}




\section{INTRODUÇÃO}

O consumo de drogas é um dos principais problemas sociais e de saúde pública em todo o mundo, acarretando potenciais prejuízos de ordem física, psíquica e social ao usuário e à segurança da sociedade, principalmente por sua associação com a violência, a criminalidade e a marginalidade $[1,2,34]$.

De acordo com o Relatório anual de 2018, sobre o uso de drogas no mundo, elaborado pelo Escritório das Nações Unidas sobre Drogas e Crime (UNODC), um quarto de bilhão de pessoas, o equivalente a $5,5 \%$ da população adulta mundial com idade entre 15 e 64 anos, consumiram drogas pelo menos uma vez em 2017. Estima-se que 13\% desses usuários, sofrem com os efeitos tóxicos do uso abusivo de substâncias psicoativas, incluindo a dependência, e necessitam de tratamento. No entanto, a disponibilidade e o acesso a serviços de tratamento permanecem limitados; apenas uma em cada seis pessoas recebe intervenção a cada ano $[5,6]$.

As drogas ilícitas são substâncias que, por seu risco à saúde, têm sua comercialização e consumo proibidos por lei [7]. Mundialmente, a droga ilícita mais consumida é a maconha $[8,9]$.

Os resultados da pesquisa nos Estados Unidos desenvolvida pela Substance Abuse and Mental Health Services Administration (SAMHSA), realizada em 2017, sobre uso de drogas e saúde, demostraram que 30,5 milhões de americanos com doze anos ou mais usaram drogas ilícitas nos últimos trinta dias e que 1 em cada 4 jovens adultos (18 - 25 anos) eram usuários dessas substâncias. Estima-se que desses 30,5 milhões de pessoas 26,0 milhões eram usuários de maconha, 2,2 milhões eram usuárias de cocaína e seus derivados e 3,2 milhões eram usuários de analgésicos ou tranquilizantes prescritos. $\mathrm{O}$ aumento no consumo de maconha observado nesse ano foi atribuído ao crescimento no consumo dessa substância por jovens adultos de 18 a 25 anos e adultos com 26 anos ou mais [10].

O último levantamento, de 2010, feito entre estudantes do ensino fundamental e médio das redes pública e privada das capitais brasileiras, os solventes/inalantes $(5,2 \%)$, a maconha $(3,7 \%)$ e os ansiolíticos $(2,6 \%)$ foram as drogas mais consumidas [11]. Padrão semelhante foi verificado entre crianças e adolescentes em situação de rua de todo o território brasileiro, sendo os solventes $(36,8 \%)$, a maconha $(32,1 \%)$ e a cocaína e derivados $(18,5 \%)$ as drogas de maior uso [12].

O mais recente levantamento brasileiro sobre o uso de drogas, realizado em 2015, abrangendo todo o território nacional, revelou que aproximadamente 15 milhões de pessoas fizeram uso de substância ilícita alguma vez na vida, sendo que, nos últimos 12 meses, a prevalência da faixa etária foi entre adultos jovens ( 25 a 34 anos). Em relação à prevalência do uso de substâncias ilícitas a maconha figurou em primeiro lugar $(7,7 \%)$, seguida da cocaína $(3,1 \%)$, crack e similares $(0,9 \%)$ [13]. O uso de maconha aumentou significativamente em menos de 10 anos, uma vez que, no levantamento nacional do ano de 2012, um total de 7,8 milhões de brasileiros adultos $(5,8 \%$ da população estudada) fez uso dessa droga pelo menos uma vez na vida. Entre os adolescentes, esse número foi de 597 mil indivíduos $(4,3 \%)$ dentre quase 14 milhões de adolescentes participantes. A cocaína classificou-se em $2^{\circ}$ lugar dentre as drogas ilícitas mais usadas no referido ano $(3,8 \%)[14]$.

As apreensões de drogas ilícitas feitas pelas Polícias Federal, Estadual e Municipal fornecem dados indiretos do consumo de drogas e permitem traçar o perfil do uso dessas substâncias em uma determinada população [15]. As reportagens que abordam apreensão de drogas também oferecem dados indiretos do consumo de drogas e permite traçar o perfil do uso dessas substâncias.

Portanto, o presente estudo teve como objetivo descrever o perfil de apreensão de drogas ilícitas na região Centro-Oeste de Minas Gerais, Brasil, comparando as informações disponíveis na mídia com os dados reais fornecidos pela Perícia Criminal da Polícia Civil de Divinópolis-Minas Gerais.

\section{MATERIAIS E MÉTODOS}

\subsection{Tipo de pesquisa}

Trata-se de um estudo observacional descritivo, realizado através de reportagens que abordassem apreensões de drogas em cidades pertencentes ao CentroOeste de Minas Gerais publicadas no período de janeiro de 2017 a dezembro de 2018 e de dados dos Registros de Eventos da Defesa Social (REDS) sobre apreensões de drogas nos anos de 2017 e 2018, fornecidos pela Perícia Criminal da Polícia Civil de Divinópolis-Minas Gerais.

\subsection{Coleta de dados}

A coleta das reportagens foi realizada entre 19 de novembro de 2018 e 02 de fevereiro de 2019. A busca foi feita na plataforma de pesquisa eletrônica Google.com e os descritores foram: "Apreensão", "Drogas", "Cidade-MG", substituindo pelas cidades pertencentes à região CentroOeste de Minas Gerais. E em seguida, análise dos REDS envolvendo drogas, entre o período de 2017 e 2018.

As cidades foram agrupadas de acordo com a Delegacia Regional (DR) responsável, sendo:

- DR de Divinópolis: Divinópolis, Carmo do Cajuru, Itaúna, Itatiaiuçu, Cláudio; 
- DR de Pará de Minas: Pará de Minas, Papagaios, Onça do Pitangui, São Gonçalo do Pará, Igaratinga, São José da Varginha, Maravilhas, Pequi;

- DR de Nova Serrana - Nova Serrana, Araújos, Perdigão, Conceição do Pará, Leandro Ferreira, Pitangui;

- DR de Formiga: Formiga, Arcos, Itapecerica, Pimenta, Tapiraí, Bambuí, Camacho, Iguatama, Medeiros, Pains, São Sebastião do Oeste, Córrego Fundo;

- DR de Bom Despacho: Bom Despacho, Pompéu, Lagoa da Prata, Abaeté, Biquinhas, Martinho Campos, Morada Nova, Paineiras Monte, Quartel Geral, Córrego Danta, Dores do Indaiá, Estrela do Indaiá, Japaraíba, Luz, Pedra do Indaiá, Serra da Saudade, Santo Antônio do Monte, Cedo do Abaeté, Moema.

\subsection{Tipo de pesquisa}

As variáveis analisadas foram quantitativas e qualitativas e relacionam-se com o indivíduo (sexo e idade), bem como com o fato ocorrido (local do fato, tipo de droga apreendida e quantidade de droga apreendida), quantidade de registros relacionados ao consumo e tráfico de drogas.

\subsection{Análises Estatísticas}

Os dados para o estudo foram coletados por meio de banco de dados elaborado no programa Questionnaire Development System ${ }^{\circledR}$ (versão 2.6.1) e posteriormente exportados para o programa estatístico Statistical Package for the Social Sciences (SPSS) versão 19.0, para as análises estatísticas.

Para caracterização do perfil de apreensão de drogas da região Centro-Oeste de Minas Gerais realizou-se análise descritiva das variáveis. Elas foram representadas por meio de distribuição de frequência, mediana, intervalo interquartil, média e desvio padrão. Para as variáveis numéricas realizou-se o teste de Kolmogorov-Smirnov a fim de verificar a normalidade dos dados. Entretanto esse teste não foi realizado, para as seguintes drogas e suas respectivas formas: cocaína em peso e tablete; crack em tablete; folha de coca em peso; haxixe em porção e loló em frascos devido ao número de dados insuficientes.

\section{RESULTADOS E DISCUSSÃO}

Ao realizar a análise das 98 reportagens disponíveis acerca das apreensões de drogas na região Centro-Oeste de Minas Gerais, no período entre os anos de 2017 a 2018, foi possível identificar um total de 208 indivíduos detidos por envolvimento com o tráfico de drogas.
Entre todos os indivíduos detidos durante as apreensões de drogas, a maioria era do sexo masculino $(\mathrm{n}=152 /$ $73,1 \%)$ e apenas $10,6 \%(n=22)$ do sexo feminino. Os dados ausentes, em que não foram encontradas informações sobre o sexo do indivíduo, representam 16,3\% $(n=22)$. Dados semelhantes foram encontrados pelo Instituto de Segurança Pública (ISP), o número de detidos do sexo masculino $(\mathrm{n}=27.250)$ é muito maior que os do sexo feminino $(n=2.199)$ [16].

Ao considerar a idade de todos os indivíduos detidos, observou-se que a idade mínima do estudo foi de 14 anos e a máxima de 57 anos, e que a mediana da idade é de 21 anos com intervalo interquartil $(25 \%$ e $75 \%)$ de 18 e 26,5 anos. Para o sexo feminino, a idade mínima encontrada foi de 16 anos e a máxima de 38 anos, sendo a média das idades de 24,75 anos com desvio padrão de 5,54. Para o sexo masculino, a idade mínima encontrada foi de 14 anos e a máxima de 57 anos com mediana de 21 anos e intervalo interquartil (25\% e 75\%) de 17 e 26 anos. A UNODC mostra que o uso de drogas é maior entre os jovens que entre idosos e que a prevalência é maior entre indivíduos com idade entre 18 e 25 anos. Dados apresentados pelo Instituto de Segurança Pública (ISP) mostram que a maioria dos detidos por envolvimento com drogas são adolescentes e jovens menores de 21 anos [16,17].

Constatou-se, também, que dentre todos os indivíduos detidos durante as apreensões de drogas, a maioria é maior de idade $(\mathrm{n}=124 / 59,6 \%)$ e apenas $17,8 \%$ são menores de idade. Os dados ausentes, em que não foram encontradas informações sobre a idade do indivíduo, representam $22,6 \%(n=47)$. Segundo o ISP, os detidos menores de idade representam aproximadamente um quarto do total de detidos [16].

Foi possível identificar um total de 101 apreensões de drogas efetuadas entre o período de 2017 a 2018 de acordo com as reportagens. Para cada uma dessas apreensões foram detidos e apreendidos em média, respectivamente, dois indivíduos e dois diferentes tipos de drogas. Conforme a literatura, as apreensões de drogas podem ser analisadas em termos de quantidade de droga apreendida ou por meio do número de apreensões. E se consideradas em conjunto, alterações no número de apreensões e de quantidades apreendidas, podem auxiliar na identificação de padrões de oferta de drogas [18].

A maioria das apreensões de drogas ocorreram na Regional de Formiga ( $\mathrm{n}=39 / 38,61 \%$ ) seguido da Regional de Bom Despacho $(n=31 / 30,69 \%)$, Pará de Minas $(n=28 / 27,72 \%)$, Divinópolis $(n=2 / 1,98 \%)$ e Nova Serrana $(n=1 / 0,99 \%)$, tendo como cidades de maiores apreensões Bambuí ( $n=8)$ e Formiga ( $n=7)$; Lagoa da Prata $(n=5)$, Bom Despacho $(n=4)$ e Luz $(n=4)$; Papagaios $(n=5)$ e Pequi $(\mathrm{n}=5) ; \quad$ Divinópolis $\quad(\mathrm{n}=2) ; \quad$ Nova Serrana $\quad(\mathrm{n}=1)$ respectivamente (Figura 1). Os números de apreensões de drogas ilícitas nas cidades foram inferiores ao esperado e o 
principal motivo dessa limitação foi o reduzido número de reportagens disponíveis que atendiam às condições previamente estipuladas para a coleta de dados. Tendo em vista que não são todas as apreensões de drogas que são publicadas. $\mathrm{Na}$ busca das reportagens disponíveis no período de janeiro de 2017 a dezembro de 2018, no mês de janeiro de 2017 não foi encontrado nenhuma reportagem que abordasse apreensão de drogas nas cidades selecionadas pelo presente estudo.

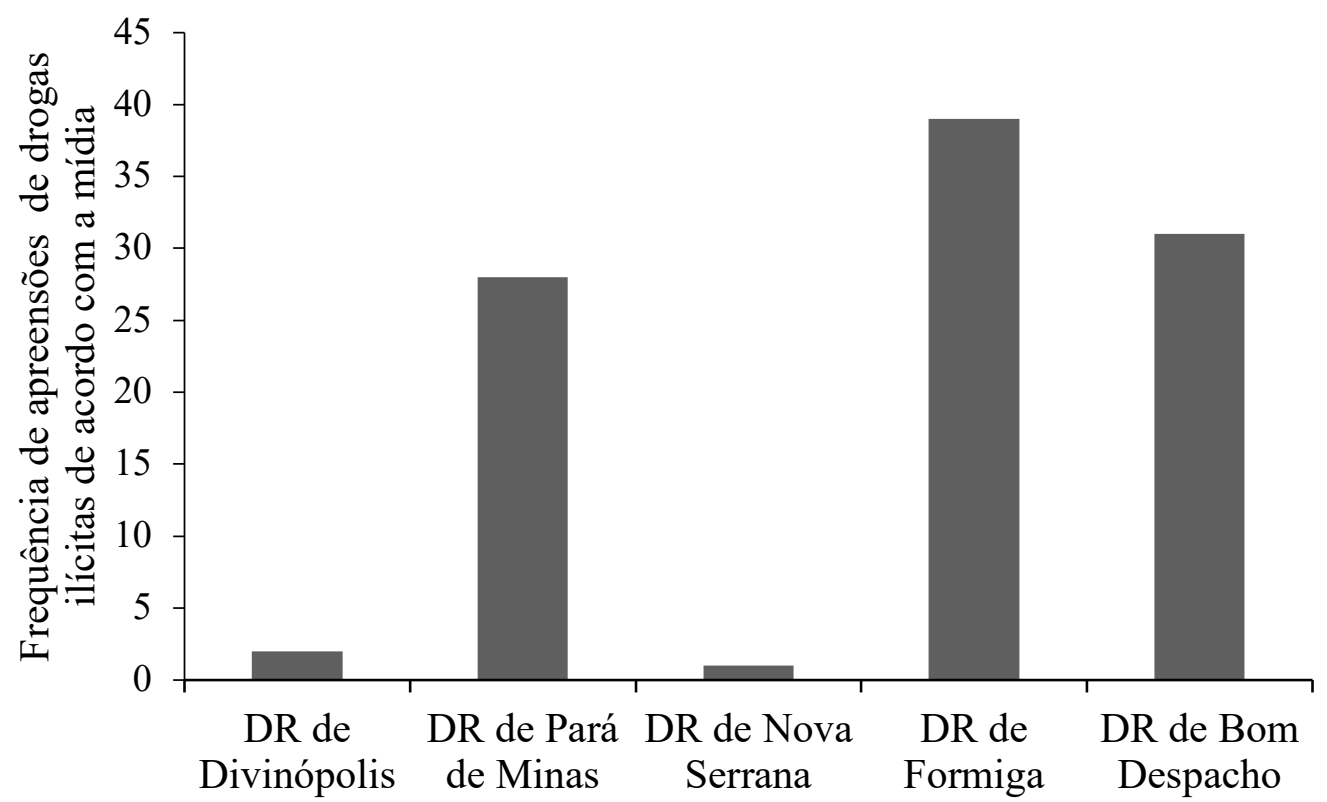

Figura 1. Frequência das cidades da região Centro-Oeste de Minas Gerais que houve apreensão de drogas ilícitas de acordo com a mídia entre 2017 a 2018. (DR) Delegacia Regional

Quando comparado os dados anteriores com os dados reais, fornecidos pela Perícia Criminal da Polícia Civil de Minas Gerais (Figura 2) é possível constatar que há uma diferença significativa entre as cidades com maiores de frequência de apreensões de drogas ilícitas.

Apesar de as maiores apreensões de drogas serem atribuídas pelas reportagens as Regionais de Formiga, Bom Despacho, Pará de Minas, Divinópolis e Nova Serrana, elas não estão entre as cidades com maiores apreensões. Segundo as informações reais da Perícia Criminal, as regionais de Nova Serrana $(n=18.347 / 41,38 \%)$, Pará de Minas ( $n=8.665 / 19,54 \%)$, Divinópolis $(n=6.394 / 14,42 \%)$, Bom Despacho $\quad(\mathrm{n}=6.303 / 14,22 \%), \quad$ Formiga $(n=4.627 / 10,44 \%)$. Em relação as cidades com as maiores apreensões de cada Regional, estão: Araújos ( $n=9.083)$ e Conceição do Pará $(n=6.411)$; Pará de Minas $(n=6.852)$ e São Gonçalo do Pará $(n=795)$; Cláudio $(n=4.152)$ e Divinópolis $(\mathrm{n}=1.372)$; Luz $(\mathrm{n}=1.720)$ e Santo Antônio do Monte $(n=1.079)$; Pimenta $(n=1.271)$ e Medeiros $(\mathrm{n}=1.262)$. 


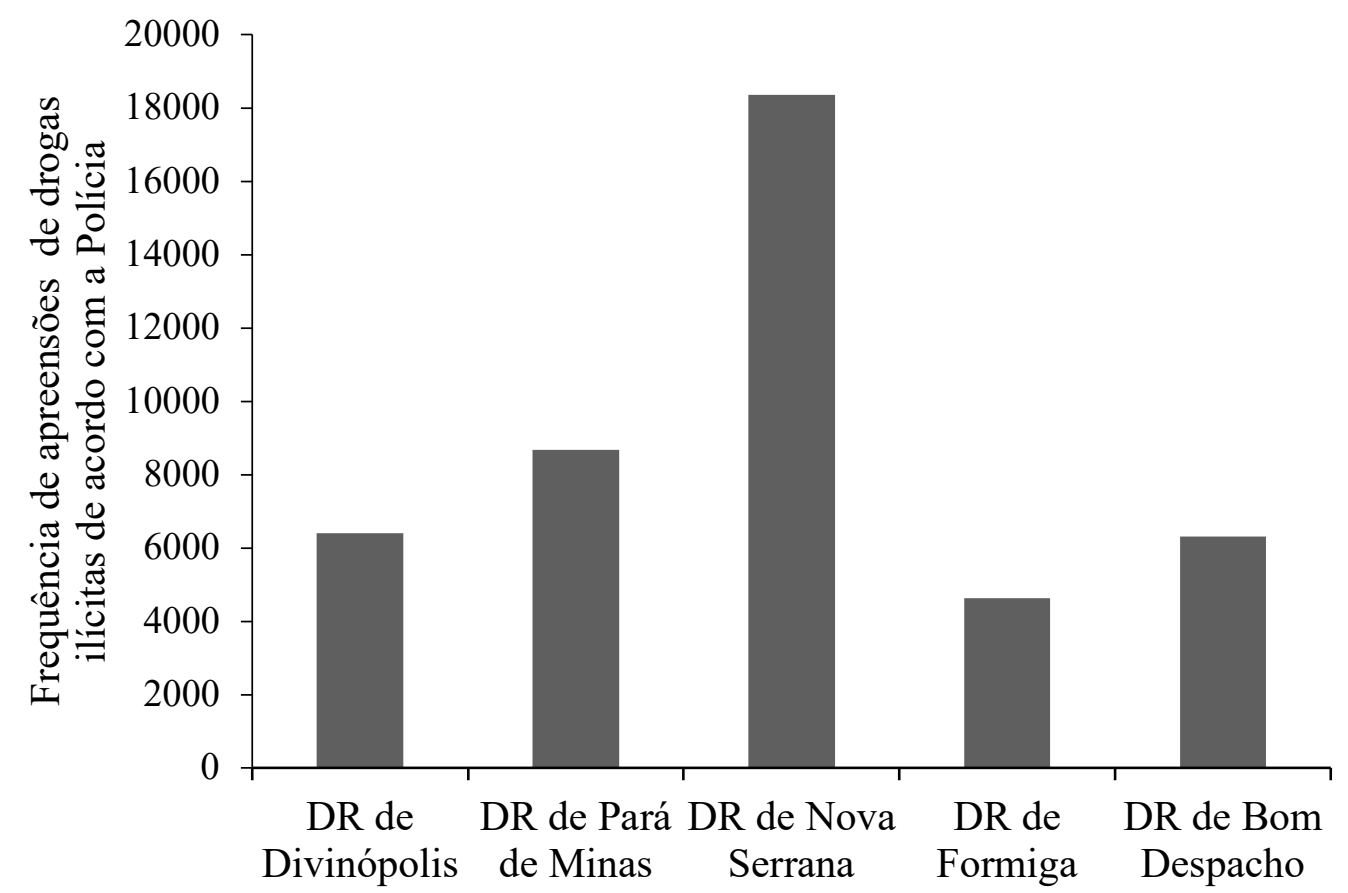

Figura 2. Frequência das cidades da região Centro-Oeste de Minas Gerais que houve apreensão de drogas ilícitas de acordo com a Polícia entre 2017 a 2018. (DR) Delegacia Regional.

Ao considerar todas as apreensões de drogas, observouse que as drogas encontradas foram maconha $(\mathrm{n}=$ $75 / 74,3 \%)$, cocaína $(n=39 / 38,6 \%)$, crack $(n=37 / 36,6 \%)$, pasta base de cocaína $(n=4 / 3,96 \%)$, mudas de maconha ( $n$ $=3 / 2,97 \%)$, ecstasy $(n=3 / 2,97 \%)$, haxixe $(n=2 / 1,98 \%)$, folha de coca $(\mathrm{n}=1 / 0.99 \%)$, e loló $(\mathrm{n}=1 / 0,99 \%)$ (Figura $3)$.

De forma semelhante, uma pesquisa feita no munícipio de Itajubá, região Sul de Minas, demonstrou que a droga ilícita mais apreendida na região foi a maconha [19]. Segundo o levantamento mundial, feito em 2016, a maconha é a droga mais apreendida, seguida pela cocaína e seus derivados, opioides, substâncias psicoativas e anfetamínicos [18]. Em 2016, dois terços do total global de apreensões de maconha, foram confiscadas em países das Américas sendo que o Brasil foi o $6^{\circ}$ país com maior quantidade de maconha apreendida [20].

As Tabelas 1 e 2 mostram a quantidade média e bruta das drogas apreendidas, em suas respectivas formas, entre fevereiro de 2017 a dezembro de 2018, com base nas informações obtidas pelas reportagens analisadas. A Cannabis, popularmente conhecida como maconha, é uma substância de cor esverdeada produzida por meio da secagem e mistura de caule, sementes e folhas, e pode ser administrada na forma de cigarro ou adicionada aos alimentos. O haxixe, resina de Cannabis, é a secreção seca

de coloração preta ou marrom, dos brotos floridos da planta. A cocaína é um pó cristalino fino de cor branca ou esbranquiçada, é extraída das folhas da planta de coca e nas primeiras etapas de sua produção é possível obter substâncias como a pasta de coca. O crack ou base da cocaína apresenta-se como pequenos fragmentos irregulares (pedras) de um sólido de cor esbranquiçada. O ecstasy é um estimulante psicoativo produzido em laboratórios ilegais, geralmente é distribuído na forma de comprimidos, mas também pode-se apresentar na forma de pó ou cápsula. Os solventes, administrados por inalação, são substâncias químicas voláteis como cola, acetona, tintas [21, 22].

De modo geral, como pode ser visto na Figura 3, independente da forma em que a droga se apresenta, a maconha é a droga apreendida em maior quantidade, seguida pela cocaína e seus derivados (pasta, crack). Dados similares foram apresentados pela Polícia Rodoviária Federal do Brasil (PRF), que de janeiro a junho de 2018 aprendeu $144.000 \mathrm{~kg}$ de maconha, $8.500 \mathrm{~kg}$ de cocaína, 31.262 unidades de ecstasy e $750 \mathrm{~kg}$ de crack, e pela Polícia Federal do Brasil, que no ano de 2016, aprendeu $39.311 \mathrm{~kg}$ de cocaína e seus derivados (pasta base, crack), $224.829 \mathrm{~kg}$ de maconha e 490.270 unidades de ecstasy $[23,24]$. 


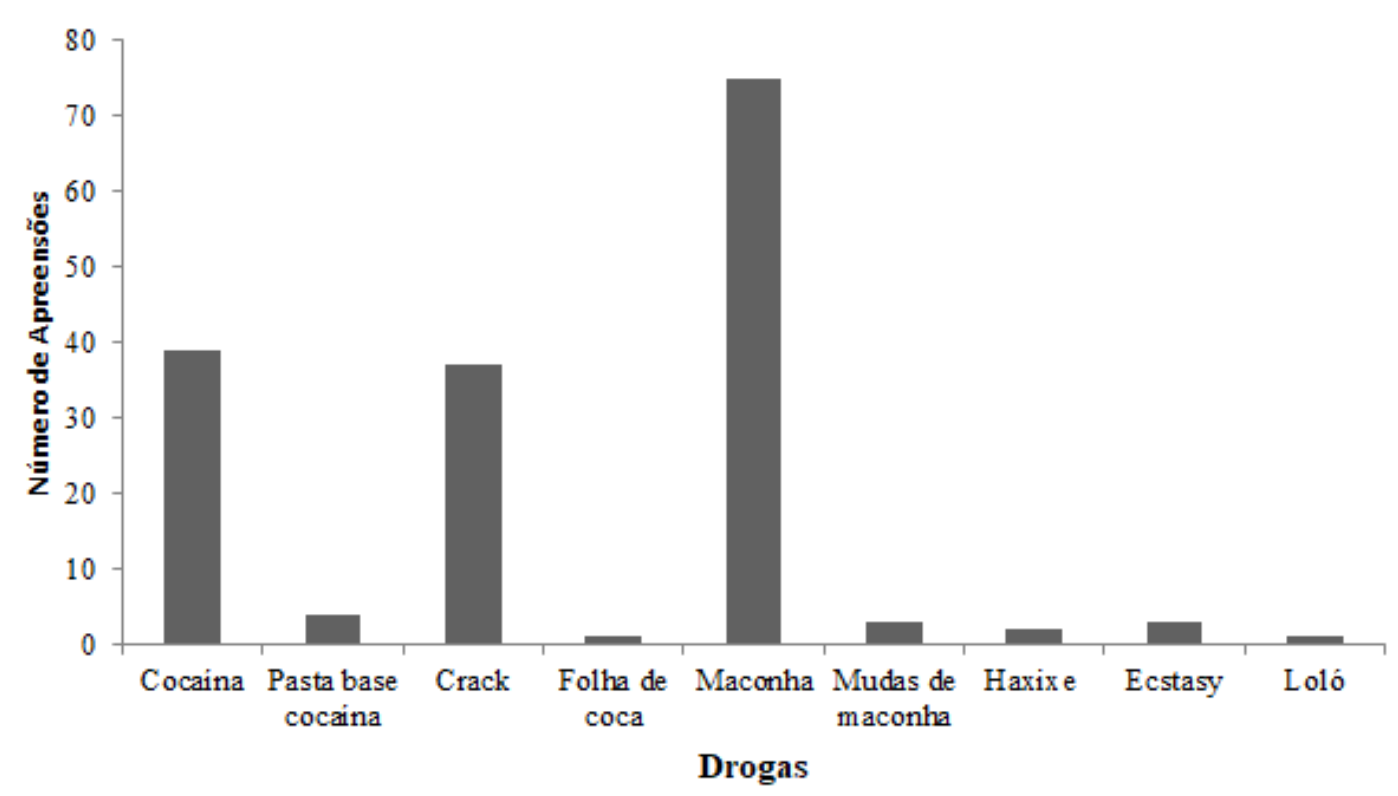

Figura 3. Frequência das drogas apreendidas na região Centro-Oeste de Minas Gerais entre o período de 2017 a 2018.

Tabela 1. Drogas apreendidas na região Centro-Oeste de Minas Gerais entre o período 2017 a 2018, segundo a mídia.

\begin{tabular}{|c|c|}
\hline DROGA & QUANTIDADE MÉDIA DE DROGA APREENDIDA \\
\hline \multicolumn{2}{|l|}{ Cocaína } \\
\hline Papelote (unidades) & $2,0(1,0 ; 16,2)^{\mathrm{a}}$ \\
\hline Pino (unidades) & $19,3(20,3)^{b}$ \\
\hline Porção (unidades) & $7,5(15,3)^{\mathrm{b}}$ \\
\hline \multicolumn{2}{|l|}{ Pasta base de cocaína } \\
\hline Peso $(\mathrm{Kg})$ & $189,17(219,45)^{\mathrm{b}}$ \\
\hline \multicolumn{2}{|l|}{ Crack } \\
\hline Pedra (unidades) & $38,39(48,36)^{\mathrm{b}}$ \\
\hline Porção (unidades) & $1,80(0,837)^{\mathrm{b}}$ \\
\hline \multicolumn{2}{|l|}{ Maconha } \\
\hline Bucha (unidades) & $4,0(1,0 ; 19,0)^{\mathrm{a}}$ \\
\hline Cigarro (unidades) & $8,75(14,17)^{b}$ \\
\hline Peso $(\mathrm{Kg})$ & $10,0(1,13 ; 57,5)^{\mathrm{a}}$ \\
\hline Porção (unidades) & $8,75(14,17)^{\mathrm{b}}$ \\
\hline Tablete (unidades) & $1,0(1,0 ; 4,0)^{\mathrm{a}}$ \\
\hline \multicolumn{2}{|l|}{ Muda de maconha } \\
\hline Unidade & $3,0(2,64)^{b}$ \\
\hline Ecstasy & \\
\hline Comprimidos (unidade) & $15,33(3,51)^{\mathrm{b}}$ \\
\hline
\end{tabular}

$\overline{{ }^{a}}$ Valores representados por mediana e intervalo interquartil $(25 \% ; 75 \%) .{ }^{\mathrm{b}}$ Valores representados por média e desvio padrão. 
Tabela 2. Drogas apreendidas na região Centro-Oeste de Minas Gerais entre janeiro de 2017 a dezembro de 2018, segundo a mídia.

\begin{tabular}{c|c}
\hline DROGA & QUANTIDADE BRUTA DE DROGA APREENDIDA \\
\hline Cocaína & 27 \\
\hline Peso (Kg) & 1 \\
\hline Tablete (unidades) & 1 \\
\hline Crack & 1 \\
\hline Tablete (unidades) & \\
\hline Folha de Coca & 2 \\
\hline Peso (Kg) & \\
\hline Haxixe & 20 \\
\hline Porção (unidades) & \\
\hline Loló & \\
\hline Frascos (unidades) & \\
\hline
\end{tabular}

O presente estudo apresentou as seguintes limitações: não foram todos os meses dentro do período de 2017 a 2018 que apresentaram dados disponíveis das apreensões de drogas realizadas nas cidades do Centro-Oeste de Minas Gerais. Das 50 cidades estudadas, 19 (Pains, Medeiros, Tapiraí, Carmo do Cajuru, Cláudio, Itatiaiuçu, Itaúna, Perdigão, Conceição do Pará, Pitangui, Leandro Ferreira, Araújos, Biquinhas, Quartel Geral, Martinho Campus, Japaraíba, Santo Antônio do Monte, Pedra do Indaía, Estrela do Indaiá), não foram encontradas nenhuma reportagem no site de busca Google.com e Onça do Pitangui e Pitangui não tiveram nenhum REDS feito pela Polícia. Não foi possível comparar os tipos e quantidades de drogas divulgadas pelas reportagens em relação aos dados reais por ausência de informação. Entretanto, fica evidente que nem todas as informações divulgadas, representam a situação real acerca dos registros de apreensões de drogas da região. As matérias jornalísticas tendem a expor a informação "mais conveniente", entretanto, isso pode gerar uma informação e/ou conhecimento equivocado para a população, uma vez que, os meios de comunicação em massa são a primeira fonte de escolha por parte da sociedade para adquirir conhecimento $[25,26]$.

\section{CONSIDERAÇÕES FINAIS}

Conclui-se que os indivíduos detidos por envolvimento com drogas, na região Centro-Oeste de Minas Gerais, são, em sua maioria, indivíduos jovens do sexo masculino e também que, dentre as drogas apreendidas, a maconha figurou em primeiro lugar seguida pela cocaína e seus derivados. Ademais, os dados levantados acerca da apreensão de drogas demonstram a dimensão dessa problemática em uma região e permitem a sua comparabilidade em nível nacional e estadual.

A relevância deste estudo encontra-se que não há disponível na literatura artigos científicos contendo o perfil de apreensão de drogas das cidades pertencentes à região do Centro-Oeste de Minas Gerais. O perfil de apreensão de drogas de uma determinada região torna-se um referencial importante para subsidiar o planejamento de políticas públicas conforme a real demanda, visando à prevenção do comércio e ao combate ao uso dessas substâncias.

A mídia é um importante canal de comunicação para disseminação de informação que pode colaborar na educação em saúde, entretanto, essas informações precisam ser bem construídas e divulgadas de maneira transparente para a sociedade, para que haja uma maior compreensão e não descriminalização no que se refere às drogas ilícitas.

\section{AGRADECIMENTOS}

Os autores gostariam de agradecer à Universidade Federal de São João del-Rei e à Perícia Criminal da Polícia Civil de Divinópolis-MG pelo apoio e suporte, possibilitando a realização deste estudo.

O presente trabalho foi realizado com apoio da Coordenação de Aperfeiçoamento de Pessoal de Nível Superior - Brasil (CAPES) - Código de Financiamento 001.

\section{REFERÊNCIAS BIBLIOGRÁFICAS}

[1] S.R. de Andrade; A.C.C. Martins.. O valor social do proerd e a educação como política pública de combate às drogas e à violência. Biblioteca Digital de Segurança Pública, Goiás, p. 1-5, 2019. 
[2] P.V.A. Di Nápoli; B.A. Chaibub. Polícias Militares x Drogas Psicotrópicas. Biblioteca Digital de Segurança Pública, Goiás, p. 1-21, 2019.

[3] K.S. Foppe; D.R. Hammond-Weinberger; B. Subedi. Estimation of the consumption of illicit drugs during special events in two communities in Western Kentucky, USA using sewage epidemiology. Science of the Total Environmen, Amsterdã, p. 249-256, 2018.

[4] B.A.R. Singulane; N.B. Silva; L.M.A. Sartes. Histórico e Fatores Associados à Criminalidade e Violência entre Dependentes de Crack. Psico-USF, Bragança Paulista, v. 21, n. 2, p. 395-407, 2016.

[5] United Nations Office on Drugs and Crime (UNODC). World Drug Report 2017. Booklet 2, Vienna: United Nations publication, 68 p., 2017. Retirado em 02/05/2019, de

https://www.unodc.org/wdr2017/field/Booklet_2_HEAL TH.pdf.

[6] United Nations Office on Drugs and Crime (UNODC). World Drug Report 2019. Booklet 2, Vienna: United Nations publication, 80 p., 2019. Retirado em 18/07/19, de https://wdr.unodc.org/wdr2019/prelaunch/WDR19_Bookl et_2_DRUG_DEMAND.pdf

[7] J.C.S. Raposo; A.C.Q. Costa; P.A.M. Valença; P.M Zarzar; A.S. Diniz; V. Colares; C. França. Uso de drogas ilícitas e binge drinking entre estudantes adolescentes. Revista Saúde Pública, São Paulo, v. 51, p. 1-7, 2017.

[8] P.P. Almeida; M.A.F.P. Novaes; R.A. Bressan; A.L.T. de Lacerda. Revisão: funcionamento executivo e uso de maconha. Revista Brasileira de Psiquiatria, São Paulo, v. 30, n. 1, p. 69-76, 2008.

[9] L. A. R. V. Marques; M. A. L. Lotif; E. M. R. Neto; T. S. Dantas; J. L. Soares; J. A. de Melo; P. L. D. \& Lobo. Abuso de drogas e suas consequências na saúde oral: uma revisão de literatura. Arquivo Brasileiro de Odontologia, Minas Gerais, v. 11, n. 1, p. 26-31, 2015.

[10] J. Bose; S.L. Hedden; R.N. Lipari; E. Park-Lee. Key Substance Use and Mental Health Indicators in the United States: Results from the 2017 National Survey on Drug Use and Health. Rockville, MD: Center for Behavioral Health Statistics and Quality, Substance Abuse and Mental Health Services Administration, 2018.

[11] E.L.A. Carlini; A.R. Noto; Z.V.D. Sanchez; C.M.A. Carlini; D.P. Locatelli; L.R. Abeid; T.C. Amato; E.S. Opaleye; C.S. Tondowski; Y.G. de Moura. VI Levantamento Nacional sobre o Consumo de Drogas Psicotrópicas entre Estudantes do Ensino Fundamental e Médio das Redes Pública e Privada de Ensino nas 27 Capitais Brasileiras - 2010. Brasília: Centro Brasileiro de Informações sobre Drogas Psicotrópicas - CEBRID: Universidade Federal de São Paulo - UNIFESP/Secretaria Nacional de Políticas sobre Drogas (SENAD), 503 p., 2010 .
[12] A.R. Noto; J.C.F. Galduróz; S.A. Nappo; E.A. Carlini. Levantamento nacional sobre o uso de drogas entre crianças e adolescentes em situação de rua nas 27 capitais brasileiras - 2003. São Paulo: Centro Brasileiro de Informações Sobre Drogas Psicotrópicas (CEBRID), 249 p., 2003.

[13] F.I.P.M.V. Bastos; M.T.L. Boni; R.B. Reis; N.B. Coutinho, C.F. Souza. III Levantamento Nacional sobre o uso de drogas pela população brasileira. Rio de Janeiro: FIOCRUZ/ICICT, 2017. 528 p.

[14] R. Laranjeira; C.S. Madruga; I. Pinsky; R. Caetano; S.S. Mitsuhiro. II Levantamento nacional de álcool e drogas (LENAD) - 2012. São Paulo: Instituto Nacional de Ciência e Tecnologia para Políticas Públicas de Álcool e Outras Drogas - INPAD/Universidade Federal de São Paulo - UNIFESP, 85p., 2014.

[15] P.C.A.V. Duarte; M.L.O.S. Formigoni. O uso de substâncias psicoativas no Brasil: módulo 1. Brasília: Secretaria Nacional de Políticas sobre Drogas, 11 ed., 146 p., 2017.

[16] Brasil. Instituto de Segurança Pública. Governo do Estado do Rio de Janeiro. (2019). Retirado em 17/05/19, de http://www.isp.rj.gov.br/

[17] United Nations Office on Drugs and Crime (UNODC). World Drug Report 2018. Booklet 4, Vienna: United Nations publication, 62 p., 2018c. Retirado em: 08/05/19,

de https://www.unodc.org/wdr2018/prelaunch/WDR18_Boo klet_4_YOUTH.pdf

[18] United Nations Office on Drugs and Crime (UNODC). World Drug Report 2018. Booklet 2, Vienna: United Nations publication, 68 p., 2018a. Retirado em 02/05/19, https://www.unodc.org/wdr2018/prelaunch/WDR18 Boo klet_2_GLOBAL.pdf

[19] N.T. Silva; M.E.M. Cardoso; D.O. Lopes; A.S. Silva; F.V. Lacerda; L.H.S. Oliveira; V.V.Souza. Prevalência de drogas aprendidas em um município do sul de Minas Gerais: análise de variáveis interferentes e propostas para a prevenção. Revista da Universidade Vale do Rio Verde, Minas Gerais, v. 14, n. 1, p. 707-713, 2016.

[20] United Nations Office on Drugs and Crime (UNODC). World Drug Report 2018. Booklet 3, Vienna: United Nations publication, 76 p., 2018b. Retirado em 08/05/19, https://www.unodc.org/wdr2018/prelaunch/WDR18_Boo klet 3_DRUG_MARKETS.pdf

[21] Departamento de Justiça dos Estados Unidos. Administração de Repressão às Drogas. Drogas de Abuso. Estados Unidos, 94 p., 2017. Retirado em 08/05/19, de https://www.dea.gov/documents/2017/06/15/drugs-abuse [22] Escritório das Nações Unidas sobre Drogas e Crimes (UNODC). Campanha global sobre drogas. $2019 \mathrm{~b}$. Retirado em 08/05/19, de 
https://www.unodc.org/documents/lpo-

brazil/Topics_drugs/Campanha-global-sobre-

drogas/getthefacts11_PT_.pdf

[23] H.M. Modesto Filho; B.A. Chaibub. Apreensão de drogas pela polícia militar do estado de goiás nos últimos anos. Biblioteca Digital de Segurança Pública Goiás, 2018.

[24] BRASIL. Ministério da Justiça e Segurança Pública. Polícia Federal. Estatística de drogas apreendidas. (2017).

Retirado em 08/03/2018, de http://www.pf.gov.br/imprensa/estatistica/drogas

[25] C.R.A. Medeiros; V.M. Medeiros; M.I.G. Conceição. Mídia advocacy: alternativa de prevenção ao uso de drogas. Divulgação Científica e Tecnologica IFPB, v. 27, p. 72-80, 2015.

[26] R. Figueiredo; M. Feffermann; R. Adorno. Drogas \& sociedade contemporânea: perspectivas para além do Proibicionismo. São Paulo: Instituto de Saúde, 356 p., 2017.

\section{APÊNDICE: REPORTAGENS ANALISADAS NESTE ESTUDO}

Morada Nova de Minas: Adolescente é detido com droga e réplica de arma. Disponível em: https://grnews.com.br/05102017/radar-policial/moradanova-de-minas-adolescente-e-detido-com-droga-e-replicade-arma

\footnotetext{
Morada Nova de Minas - PM prende autor de tráfico de drogas. Disponível em https://www.policiamilitar.mg.gov.br/portal$\mathrm{pm} / 7 \mathrm{rpm} /$ conteudo.action? conteudo $=180454 \&$ tipoConteu do $=$ noticia
}

Acusados de tráfico de drogas são presos em flagrante. Disponível em: https://www.nossojornalabaete.com.br/2018/01/29/acusad os-de-trafico-de-drogas-sao-presos-em-flagrante/

PM prende traficante com quase 10 mil em Abaeté após denuncias feitas ao 181. Disponível em: https://g1.globo.com/mg/centro-oeste/noticia/pm-prendetraficante-com-quase-r10-mil-em-abaete-apos-denunciasfeitas-ao-181.ghtml

Em Abaeté grupo é detido com drogas em táxi. Disponível em: https://gl.globo.com/mg/centrooeste/noticia/em-abaete-grupo-e-detido-com-drogas-emtaxi.ghtml

Paineiras: Casal é preso com maconha, cocaína, dinheiro e réplica de arma. Disponível em: https://grnews.com.br/12122017/radar-policial/paineiras- casal-e-preso-com-maconha-cocaina-dinheiro-e-replicade-arma

Paineiras: Suspeito de tráfico de drogas é preso com maconha, dinheiro e celulares. Disponível em: https:/grnews.com.br/18052017/radar-policial/paineirassuspeito-de-trafico-de-drogas-e-preso-com-maconhadinheiro-e-celulares

$7^{\circ}$ BPM prende traficante em Cedro do Abaeté. Disponível em: https://www.policiamilitar.mg.gov.br/portal$\mathrm{pm} / 7 \mathrm{rpm} /$ conteudo.action?conteudo $=167220 \&$ tipoConteu do $=$ noticia

PM prende autor de tráfico de drogas e recaptura dois foragidos da justiça em Serra da Saudade. Disponível em: https://www.destaknewsbrasil.com.br/index.php/2018/06/ 13/pm-prende-autor-de-trafico-de-drogas-e-recapturadois-foragidos-da-justica-em-serra-da-saudade-mg/

Operação Limpa Trilho é feita em Pompéu para combate ao tráfico de drogas. Disponível em: https://g1.globo.com/mg/centro-oeste/noticia/operacaolimpa-trilho-e-feita-em-pompeu-para-combate-ao-traficode-drogas.ghtml

PM aprende drogas com autores que praticavam direção perigosa em Pompéu. Disponível em: https://www.destaknewsbrasil.com.br/index.php/2018/11/ 25/pm-apreende-drogas-com-autores-que-praticavamdirecao-perigosa-em-pompeu-mg/

PM aborda individuo suspeito e apreende arma e drogas em Pompéu. Disponível em: https://www.destaknewsbrasil.com.br/index.php/2018/12/ 28/pm-aborda-individuo-suspeito-e-apreende-arma-edrogas-em-pompeu-mg/

Tabletes de maconha são apreendidos dentro de residência em Dores do Indaiá. Disponível em: https://www.g37.com.br/c/policial/tabletes-de-maconhasao-apreendidos-dentro-de-residencia-em-dores-do-indaia

Dores do Indaiá - durante cumprimento de mandato, PM prende quadrilha por tráfico de drogas. Disponível em: https://www.policiamilitar.mg.gov.br/portal$\mathrm{pm} / 7 \mathrm{rpm} /$ conteudo.action?conteudo $=180912 \&$ tipoConteu do $=$ noticia

Cinco adolescentes são apreendidos transportando drogas em Dores do Indaiá. Disponível em: https://www.tapiraimgtv.com.br/noticia/4697/Cinco- 
adolescentes-sao-apreendidos-transportando-drogas--emDores-do-Indaia

Bom Despacho: família inteira é presa por tráfico de drogas. Disponível em: https://grnews.com.br/16032018/radar-policial/bomdespacho-familia-inteira-e-presa-por-trafico-de-drogas

Jovens são presos com 60 quilos de maconha na BR 262 em Bom Despacho. Disponível em: https://www.otempo.com.br/cidades/jovens-s\%C3\%A3opresos-com-60-quilos-de-maconha-na-br-262-em-bomdespacho-1.2073627

Homem é preso com 27 kg de cocaína na BR - 262 em Bom Despacho. Disponível em: https://www.em.com.br/app/noticia/gerais/2018/10/21/int erna_gerais,998938/homem-e-preso-com-27-kg-decocaina-na-br-262-em-bom-despacho.shtml

Pasta base de cocaína é apreendida em caminhão na BR - 262 em Bom Despacho. Disponível em: https://g1.globo.com/mg/centrooeste/noticia/2018/12/30/pasta-base-de-cocaina-eapreendida-em-caminhao-na-br-262-em-bomdespacho.ghtml

Jovem é detido com droga e canivete dentro de sala de aula em Córrego Danta. Disponível em: https://g1.globo.com/mg/centro-oeste/noticia/jovem-edetido-com-droga-e-canivete-dentro-de-sala-de-aula-emcorrego-danta-mg.ghtml

Polícia Civil apreende mais de 10 quilos de maconha e cocaína a caminho de Patos de Minas. Disponível em: https:/www.patosnoticias.com.br/noticia/20390-policiacivil-apreende-mais-de-10-quilos-de-maconha-e-cocainaa-caminho-de-patos-de-minas

PM aprende drogas em residência no município de Luz MG. Disponível em: https://www.sistemampa.com.br/noticias/policial/pmapreende-drogas-em-residencia-no-municipio-de-luz-mg/

Polícia apreende $10 \mathrm{~kg}$ de maconha dentro de ônibus intermunicipal na MG - 176 em Luz. Disponível em: https:/g1.globo.com/mg/centro-oeste/noticia/policiaapreende-10-kg-de-maconha-dentro-de-onibusintermunicipal-na-mg-176-em-luz.ghtml

Policia Militar prende mulher suspeita de traficar drogas em Luz. Disponível em: https://www.destaknewsbrasil.com.br/index.php/2018/07/ 09/policia-militar-prende-mulher-suspeita-de-traficardrogas-em-luz-mg/

Moema: traficantes dão presos com droga e dinheiro. Disponível em: https:/grnews.com.br/16112017/radarpolicial/moema-traficantes-sao-presos-com-droga-edinheiro

Oito pessoas são presas por tráfico de drogas durante operação policial em Moema. Disponível em: https:/www.jornalcidademg.com.br/oito-pessoas-saopresas-por-trafico-de-drogas-durante-operacao-policialem-moema/

Homem de 23 anos é preso suspeito de tráfico de drogas em Lagoa da Prata. Disponível em: https://www.g37.com.br/c/policia/homem-de-23-anos-epreso-suspeito-de-trafico-de-drogas-em-lagoa-da-prata

Homem é preso e 11 buchas de maconha são apreendidas em Lagoa da Prata. Disponível em: https://www.ultimasnoticias.inf.br/noticia/homem-epreso-e-11-buchas-de-maconha-sao-apreendidas-emlagoa-da-prata/

PM apreende grande quantidade de drogas em Lagoa da Prata. Disponível em: https://www.ultimasnoticias.inf.br/noticia/pm-apreendegrande-quantidade-drogas-em-lagoa-da-prata/

Dupla é detida por suspeita de tráfico de drogas em Lagoa da Prata. Disponível em: https:/g1.globo.com/mg/centro-oeste/noticia/dupla-edetida-por-suspeita-de-trafico-de-drogas-em-lagoa-daprata.ghtml

PM prende trio por tráfico de drogas em Lagoa da Prata. Disponível em: https:/www.destaknewsbrasil.com.br/index.php/2018/11/ 28/pm-prende-trio-por-trafico-de-drogas-em-lagoa-daprata-mg/

PM prende trio por tráfico de drogas em Papagaios. Disponível em: https:/www.destaknewsbrasil.com.br/index.php/2018/07/ 01/pm-prende-trio-por-trafico-de-drogas-em-papagaios$\mathrm{mg} /$

PM apreende crack e maconha com jovens em Papagaios. Disponível em: http://www.jcnoticias.com.br/jc-noticias/policial/pmapreende-crack-e-maconha-com-jovens-em-papagaios 
Também em Papagaios a polícia deteve 4 pessoas e apreendeu maconha e dinheiro. Disponível em: http://www.santacruzam.com/policial/noticia/45534/0

Adolescente é apreendido com drogas em Papagaios. Disponível

https://www.g37.com.br/c/policia/adolescente-e-

apreendido-com-drogas-em-papagaios

PM apreende drogas, arma e munições com adolescente em Papagaios. Disponível em https://g1.globo.com/mg/centro-oeste/noticia/pmapreende-drogas-arma-e-municoes-com-adolescente-empapagaios-mg.ghtml

Suspeito de tráfico de drogas é presos com cocaína durante batida policial em Maravilhas. Disponível em: https://grnews.com.br/30042018/radar-policial/suspeitode-trafico-de-drogas-e-preso-com-cocaina-durante-batidapolicial-em-maravilhas

PM faz apreensão em Papagaios e Maravilhas. Disponível em: http://totalfm.com.br/pm-faz-apreensaoem-papagaios-e-maravilhas/

Operação da PM apreende armas, drogas e prende grupo em Maravilhas - MG. Disponível em: https://g1.globo.com/mg/triangulo-

mineiro/noticia/operacao-da-pm-apreende-armas-drogase-prende-grupo-em-maravilhas-mg.ghtml

19 CIA PM IND - PM apreende drogas durante Operação Esmeraldas - 40 Anos da $7^{\mathrm{a}}$ Região PM. Disponível em: https://www.policiamilitar.mg.gov.br/portal$\mathrm{pm} / 7 \mathrm{rpm} /$ conteudo.action? conteudo $=138819 \&$ tipoConteu do $=$ noticia

19 CIA PM IND - PM apreende drogas durante Operação Esmeraldas - 40 Anos da $7^{\mathrm{a}}$ Região PM. Disponível em: https://www.policiamilitar.mg.gov.br/portal$\mathrm{pm} / 7 \mathrm{rpm} /$ conteudo.action? conteudo $=138819 \&$ tipoConteu do=noticia

PM apreende 188 pedras de crack e 59 pinos de cocaína com dupla de infratores em Pequi - MG. Disponível em: https://www.destaknewsbrasil.com.br/index.php/2018/04/ 14/pm-apreende-188-pedras-de-crack-e-59-pinos-decocaina-com-dupla-de-infratores-em-pequi-mg/

Pequi: suspeito de trafico de drogas é preso com 17 buchas de maconha. Disponível em https://grnews.com.br/02042018/radar-policial/pequi- suspeito-de-trafico-de-drogas-e-preso-com-17-buchas-demaconha

Dois são detidos com cocaína e maconha em Pequi. Disponível em: http://www.jcnoticias.com.br/jcnoticias/policial/dois-sao-detidos-com-cocaina-emaconha-em-pequi

Cinco são detidos por tráfico de drogas em Pequi. Disponível em: http://www.jcnoticias.com.br/jcnoticias/policial/cinco-sao-detidos-por-trafico-de-drogasem-pequi

Apreensão de drogas em Onça de Pitangui. Disponível em: https://www.g37.com.br/c/policial/tentativa-dehomicidio-apreensao-de-30-kg-de-maconha-e-prisao-deautor-por-trafico-de-drogas-neste-final-de-semana-emdivinopolis

Polícia Militar prende autor de tráfico de drogas no bairro Nova Fortaleza em Divinópolis. Disponível em: https://www.g37.com.br/c/policial/tentativa-dehomicidio-apreensao-de-30-kg-de-maconha-e-prisao-deautor-por-trafico-de-drogas-neste-final-de-semana-emdivinopolis

Patrulha de Operações do $23^{\circ}$ Batalhão de Polícia Militar apreende cerca de $30 \mathrm{Kg}$ de drogas em Divinópolis. Disponível em: https://www.g37.com.br/c/policial/tentativa-dehomicidio-apreensao-de-30-kg-de-maconha-e-prisao-deautor-por-trafico-de-drogas-neste-final-de-semana-emdivinopolis

Homem é preso por trafico de drogas em Onça do Pitangui. Disponível em: http://www.jcnoticias.com.br/jcnoticias/policial/homem-e-preso-por-trafico-de-drogasem-onca-de-pitangui/30

Três jovens presos em Onça do Pitangui. Disponível em: http://www.santacruzam.com/policial/noticia/48696/0

Dupla que vendia drogas pelo telefone é presa em Onça de Pitangui. Disponível em: http://www.santacruzam.com/policial/noticia/44388/60

19 CIA PM IND- São José da Varginha: PM apreende cocaína e maconha com autores de tráfico durante a festa do Tomate. Disponível em: https://www.policiamilitar.mg.gov.br/portal-

$\mathrm{pm} / 7 \mathrm{rpm} /$ conteudo.action?conteudo=140927\&tipoCo nteudo $=$ noticia 
Jovens são detidos com drogas em são José da Varginha. Disponível em: http://www.jcnoticias.com.br/jc-noticias/policial/jovenssao-detidos-com-drogas-em-sao-jose-da-varginha/7560

São José da Varginha: adolescente suspeito de trafico é apreendido com drogas. Disponível em: https://grnews.com.br/04122017/radar-policial/sao-joseda-varginha-adolescente-suspeito-de-trafico-eapreendido-com-drogas

PF apreende avião com $430 \mathrm{~kg}$ de pasta base de cocaína em MG. Disponível em: https://veja.abril.com.br/brasil/pfapreende-aviao-com-430-kg-de-pasta-base-de-cocainaem-mg/

Casal é preso suspeito de tráfico de drogas em Pará de Minas. Disponível em: https://g1.globo.com/mg/centrooeste/noticia/2018/10/29/casal-e-preso-suspeito-detrafico-de-drogas-em-para-de-minas.ghtml

60PM: Polícia Militar prende autores de tráfico de drogas no Bairro Industrial, em Nova Serrana. Disponível em: https://www.policiamilitar.mg.gov.br/portal$\mathrm{pm} / 7 \mathrm{rpm} /$ conteudo.action?conteudo=176341\&tipoConteu do $=$ noticia

Drogas e armas apreendidas em residência de São Gonçalo do Pará. Disponível em: http://www.santacruzam.com/policial/noticia/48040/84

Três são detidos com drogas e replica de submetralhadora em São Gonçalo do Pará. Disponível em: http://www.jcnoticias.com.br/jc-noticias/policial/tres-saodetidos-com-drogas-e-replica-de-submetralhadora-emsao-goncalo-do-para/0

Grupo é preso com armas, drogas e munições em São Gonçalo do Pará. Disponível em https://g1.globo.com/mg/centro-oeste/noticia/grupo-epreso-com-armas-drogas-e-municoes-em-sao-goncalo-dopara.ghtml

ROTAM apreende armas e drogas em São Gonçalo do Pará. Disponível em https://www.g37.com.br/c/policia/rotam-apreende-armase-drogas-em-sao-goncalo-do-para

19 CIA PM IND - IGARATINGA: PM prende dupla por tráfico de drogas. Disponível em: https://www.policiamilitar.mg.gov.br/portal$\mathrm{pm} / 7 \mathrm{rpm} /$ conteudo.action? conteudo $=130900 \&$ tipoConteu $\mathrm{do}=$ noticia
Mais um adolescente foi apreendido com drogas na cidade de Igaratinga. Disponível em: http://radiostilo.com/index.php/noticias/policial/mais-umadolescente-foi-apreendido-com-drogas-na-cidade-deigaratinga/pag/396? nocache $=1550268745$

Igaratinga: dois presos por envolvimento com tráfico de drogas. Disponível em: https://grnews.com.br/27032017/radar-policial/igaratingadois-presos-por-envolvimento-com-trafico-de-drogas

Policia Militar apreende 48 porções de cocaína na cidade do Camacho. Disponível em: https:/www.destaknewsbrasil.com.br/index.php/2018/04/ 14/policia-militar-apreende-48-porcoes-de-cocaina-nacidade-do-camacho-mg/

Após denúncia anônima homem é preso por tráfico de drogas no Camacho. Disponível em: https://www.destaknewsbrasil.com.br/index.php/2018/08/ 16/apos-denuncia-anonima-homem-e-preso-no-camachomg-com-35-papelotes-de-cocaina/

Policia Militar prende homem por tráfico de drogas no Camacho. Disponível

em https:/www.destaknewsbrasil.com.br/index.php/2018/07/ 09/policia-militar-prende-homem-por-trafico-de-drogasno-camacho-mg/

Policia Militar prende mulher por tráfico de drogas em Itapecerica na zona rural, arma de fogo é apreendida. Disponível em: http://www.folhadeitapecerica.com.br/noticia/301/policiamilitar-prende-mulher-por-trafico-de-drogas-emitapecerica-na-zona-rural-arma-de-fogo-e-apreendida

Homem é preso com drogas e dinheiro em Itapecerica. Disponível em: https://www.ultimasnoticias.inf.br/noticia/homem-epreso-com-drogas-e-dinheiro-em-itapecerica/

Menor é apreendido em São Sebastião do Oeste por suspeita de envolvimento com tráfico de drogas. Disponível em: http://www.portalamirt.com.br/portal$\mathrm{mpa} /$ menor-e-apreendido-em-sao-sebastiao-do-oeste-porenvolvimento-com-trafico-de-drogas/

Jovem é preso em São Sebastião do Oeste por suspeita de envolvimento com tráfico de drogas. Disponível em: https://www.sistemampa.com.br/noticias/policial/joveme-preso-em-sao-sebastiao-do-oeste-por-suspeita-deenvolvimento-com-trafico-de-drogas/ 
Maconha e cocaína são apreendidos pela Policia Militar em São Sebastião do Oeste - MG. Disponível em: https://www.g37.com.br/c/centro-oeste-de-

minas/maconha-e-cocaina-sao-apreendidos-pela-policiamilitar-em-sao-sebastiao-do-oeste-mg

Policia Militar aprende menor por tráfico de drogas em Formiga. Disponível em: http://araxanoticias.com.br/2017/05/24/policia-militarapreende-menor-por-trafico-de-drogas-em-formiga/

Formiga: PM prende três indivíduos por tráfico de drogas. Disponível em: https://grnews.com.br/18042018/radar-policial/formigapm-prende-tres-individuos-por-trafico-de-drogas

Policia Militar faz apreensão por trafico de drogas em Formiga.

$$
\text { Disponível }
$$

em: http://www.portalarcos.com.br/noticia/24818/PoliciaMilitar-faz-apreensao-por-trafico-de-drogas-em-Formiga

Policia Militar apreende grande quantidade de droga e prende $o$ autor em Formiga. Disponível em: https://www.destaknewsbrasil.com.br/index.php/2018/06/ 26/policia-militar-apreende-grande-quantidade-de-drogae-prende-o-autor-em-formiga-mg/

Em formiga menos é apreendido por tráfico de drogas. Disponível em: https://www.g37.com.br/c/policia/emformiga-menor-e-apreendido-por-trafico-de-drogas

Três são detidos suspeitos de tráfico de drogas em Formiga. Disponível https://www.g37.com.br/c/ultimas-noticias/tres-saodetidos-suspeitos-de-trafico-de-drogas-em-formiga

Polícia ambiental apreende mais de $1065 \mathrm{~kg}$ de maconha em Morro Cavado. Disponível em: https://www.ultimasnoticias.inf.br/noticia/policiaambiental-apreende-mais-1065-kg-de-maconha-emmorro-cavado/

Operação da PM resulta em apreensão de veículos e drogas em cidades do Centro-Oeste de Minas. Disponível em: https://g1.globo.com/mg/centrooeste/noticia/operacao-da-pm-resulta-em-apreensao-deveiculos-e-drogas-em-cidades-do-centro-oeste-deminas.ghtml

Policia Militar prendeu um homem e aprendeu um menor com droga e munição em Córrego Fundo. Disponível em:
Militar-prendeu-um-homem-e-apreendeu-um-menor-comdrogas-e-municao-em-Corrego-Fundo

Dois jovens são presos e um menos apreendido por tráfico de drogas em Córrego Fundo. Disponível em: https://www.ultimasnoticias.inf.br/noticia/dois-jovenssao-presos-e-um-menor-apreendido-por-trafico-dedrogas-em-corrego-fundo/

Polícia Miltar descobre esquema de roubo de carros e tráfico de drogas em Arcos. Disponível em: http://www.portalamirt.com.br/portal-mpa/policia-militardescobre-esquema-de-roubo-de-carros-e-trafico-dedrogas-em-arcos/

EM Arcos mulher é presa e adolescente apreendido com drogas. Disponível em: https://www.ultimasnoticias.inf.br/noticia/em-arcosmulher-e-presa-e-adolescente-apreendido-com-drogas/

Três jovens forma presos em Arcos por tráfico de drogas. Disponível em: https://www.g37.com.br/c/tresjovens-foram-presos-em-arcos-por-trafico-de-drogas/tresjovens-foram-presos-em-arcos-por-trafico-de-drogas

Trio é preso suspeito de tráfico de drogas no Bairro Planalto em Arcos. Disponível em: http://www.portalarcos.com.br/noticia/25660/Trio-epreso-suspeito-de-trafico-de-drogas-no-Bairro-Planaltoem-Arcos

Jovem é preso suspeito de trafico de drogas em Arcos. Disponível em: https:/g1.globo.com/mg/centrooeste/noticia/2018/10/22/jovem-e-preso-suspeito-detrafico-de-drogas-em-arcos.ghtml

PM apreende drogas em residência de adolescente de 16 anos em Iguatama - MG. Disponível em: https:/www.destaknewsbrasil.com.br/index.php/2018/06/ 16/pm-apreende-drogas-em-residencia-de-adolescente-de16-anos-em-iguatama-mg/

Homem é preso e menor apreendido por tráfico de drogas em Iguatama. Disponível em: http:/www.iguatamaagora.com.br/noticia/699/Homem-epreso-e-menor-e-apreendido-por-trafico-de-drogas-emIguatama

Homem é preso por trafico de drogas em Iguatama. Disponível em: http:/www.iguatamaagora.com.br/noticia/414/Homem-epreso-por-trafico-de-drogas-em-Iguatama http://www.portalarcos.com.br/noticia/25714/Policia- 
Policia Militar apreende arma, munições e drogas em Iguatama. Disponível

em: http://www.portalarcos.com.br/noticia/25625/PoliciaMilitar-apreende-arma--municoes-e-drogas-em-Iguatama

Dois são presos em Iguatama suspeitos por tráfico de drogas. Disponível

https://www.ultimasnoticias.inf.br/noticia/dois-saopresos-em-iguatama-suspeitos-por-trafico-de-drogas/

Em Bambuí homem de 18 anos é preso pela PM por tráfico de drogas. Disponível em: http://www.bambuinews.com.br/noticias-bambui/embambui-homem-de-18-anos-e-preso-pela-pm-por-traficode-drogas

Mulher é presa e adolescente apreendido com drogas e arma de fogo em Bambuí. Disponível em: https://www.g37.com.br/c/policia/mulher-e-presa-eadolescente-apreendido-com-drogas-e-arma-de-fogo-embambui

Polícia Militar realiza operação de combate ao tráfico de drogas em Bambuí. Disponível em: https://www.destaknewsbrasil.com.br/index.php/2018/03/ 16/policia-militar-realiza-operacao-de-combate-aotrafico-de-drogas-em-bambui-mg/

Veículo, drogas, arma branca, dinheiro e celular são apreendidos pela Policia Militar em Bambuí. Disponível em:

https://www.destaknewsbrasil.com.br/index.php/2018/03/ 10/veiculo-drogas-arma-branca-dinheiro-e-celular-saoapreendidos-pela-policia-militar-em-bambui-mg/

Em Bambuí seis são presos por tráfico de drogas. Disponível em: https://www.ultimasnoticias.inf.br/noticia/em-bambuiseis-sao-presos-por-trafico-de-drogas/

Caminhão carregado com maconha é apreendido na BR - 262. Disponível em: https://g1.globo.com/mg/centrooeste/noticia/2018/08/10/caminhao-carregado-commaconha-e-apreendido-na-br-262.ghtml
Homem é preso com grande quantidade de drogas em Bambuí. Disponível em: http://www.tvbambui.com.br/noticia/15400/Homem-epreso-com-grande-quantidade-de-drogas-em-Bambui

Veículo, drogas e celulares são apreendidos pela PM em Bambuí. Disponível em: http://www.tvbambui.com.br/noticia/14557/Veiculo-drogas-e-celulares-sao-apreendidos-pela-PM-em-Bambui

Polícia Militar prende dois homens por trafico de drogas em Pimenta - MG. Disponível em: https://www.destaknewsbrasil.com.br/index.php/2018/07/ 10/policia-militar-prende-dois-homens-por-trafico-dedrogas-em-pimenta-mg/

Jovem é presa suspeita de traficar drogas em Pimenta. Disponível em: https://www.ultimasnoticias.inf.br/noticia/jovem-e-presasuspeita-de-traficar-drogas-em-pimenta/

Jovem é preso e menos apreendido por tráfico de drogas em Pimenta. Disponível em: https://www.ultimasnoticias.inf.br/noticia/jovem-e-presoe-menor-apreendido-por-trafico-de-drogas-em-pimenta/ 